\title{
Primary pleural synovial sarcoma: a rare cause of hemorrhagic pleural effusion
}

\begin{abstract}
Primary pleural synovial sarcoma (PPSS) is a rare malignant pleural tumor comprising $<1 \%$ of all primary lung malignancies. Primary pleural mesothelioma (PPM) has many similar features that may cause a diagnostic dilemma due to overlapping clinical and histopathological features. We present the case of a young male with recurrent hemorrhagic pleural effusion without any obvious lung mass who was diagnosed with PPSS. This rare entity must be considered with a high index of suspicion while evaluating pleural tumors.
\end{abstract}

Key words: hemorrhagic, immunohistochemistry, pleural effusion, sarcoma, synovial

Adv Respir Med. 2021; 89: 60-62

\section{Introduction}

Synovial sarcomas (SS) are rare entities predominantly affecting the deep soft tissue of the upper and lower extremities, with the trunk and head-neck region being uncommon sites [1]. Primary synovial sarcomas of the pleura are rare malignant pleural tumors comprising $<1 \%$ of all primary lung malignancies [2]. It usually presents as a localized solid tumor and, less commonly, as diffuse pleural thickening. Common presenting features include chest pain, breathlessness, and hemothorax. There have been a few cases that have presented with pleural effusion. Differentiating PPSS from primary pleural mesothelioma (PPM) can be a diagnostic challenge owing to PPM's clinical and histological resemblance to PPSS. Immunohistochemistry (IHC) and molecular genetics can help differentiate the two. We hereby report a case of Primary Pleural Synovial Sarcoma (PPSS) presenting with recurrent massive hemorrhagic pleural effusion in a young male.

\section{Case report}

A 26-year-old patient who was a non-smoking laborer presented with chief complaints of breathlessness, cough, fever, and left-sided chest pain for one month. He was diagnosed by another clinic as having a tuberculous pleural effusion and was initiated on anti-tuberculous treatment with no relief. At presentation, he had marked respiratory distress but was hemodynamically stable. Chest radiograph from a posteroanterior view (Figure $1 \mathrm{~A}$ ) revealed a massive left pleural effusion which was drained to relieve the distress. Pleural fluid was hemorrhagic in appearance and a lymphocytic exudate. Contrast-enhanced computed tomogram (CECT) of the thorax showed a gross left pleural effusion with multiple pleural-based heterogeneously-enhancing irregular lesions, necrotic mediastinal lymphadenopathy, and contralateral metastatic canon-ball opacities (Figure 1B).

Transthoracic fine needle aspiration from the pleural-based mass lesion was suggestive of a poorly differentiated malignant tumor. Fiberoptic bronchoscopy showed a narrowed lingular bronchus. Bronchoalveolar lavage and brush cytology were negative for malignant cells, as were three pleural fluid samples. Multiple septations and grape-like nodules studding the parietal pleura (predominantly at the base) were visualized on thoracoscopy (Figure 1C). Thoracoscopic pleural

Address for correspondence: Mayank Mishra, All India Institute of Medical Sciences, Rishikesh, India; e-mail: virgodrmayank@gmail.com

DOI: 10.5603/ARM.a2020.0147

Received: 11.06 .2020

Copyright (C) 2021 PTChP

ISSN 2451-4934 

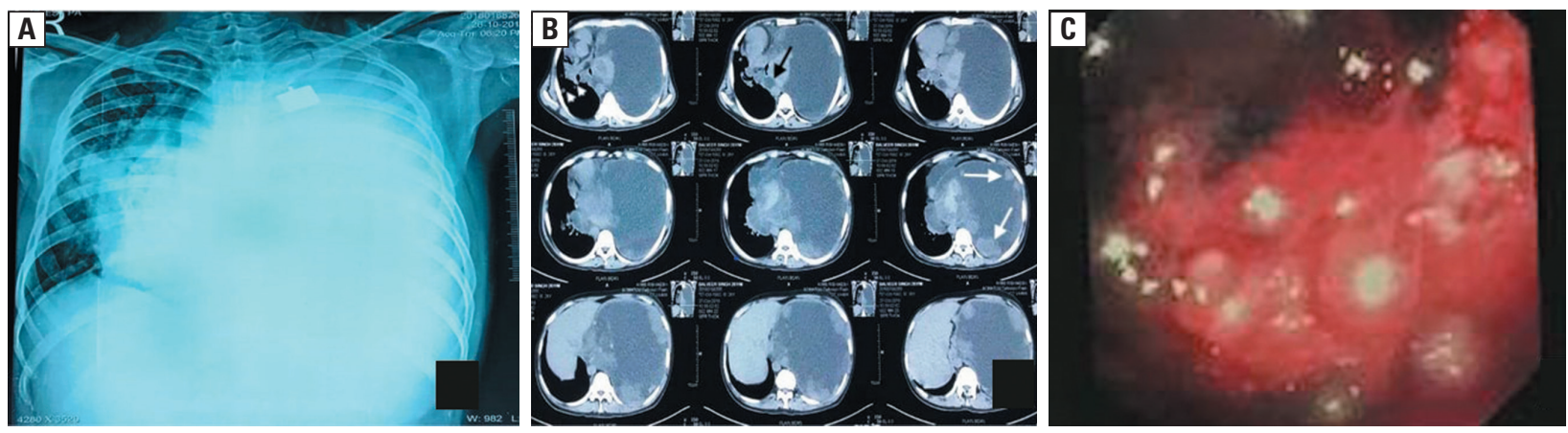

Figure 1. Chest X-ray (postero-anterior view) shows completely opaque left hemithorax with contralateral mediastinal shift (A). Contrast enhanced computed tomogram of thorax (B) shows gross left pleural effusion with multiple pleural-based irregular lesions (white arrows), necrotic mediastinal lymphadenopathy (black arrow) and contralateral metastatic canon-ball opacities (white arrowheads). Thoracoscopic image (C) showing multiple grape-like nodules studding parietal pleura

biopsy suggested a malignant small round cell tumor that was positive for TLE-1 (strong diffuse nuclear positive), CD-99 \& Pan-CK (both strong diffuse membranous positive), and synaptophysin (mild diffuse membranous positive) on IHC, suggesting a final diagnosis of PPSS (Figure 2). A bone scan showed osteoblastic lesions suggestive of skeletal metastasis in the lower one-third of the sacrum, left femur, and lateral condyle of left tibia. The patient was started on palliative doxorubicin and palliative radiotherapy.

\section{Discussion}

Synovial sarcomas are uncommon mesenchymal tumors that arise from primitive pluripotent mesenchymal cells capable of synovial differentiation. Sarcomas involving the pleural cavity include chondrosarcoma, liposarcoma, osteosarcoma, malignant schwannoma, and synovial sarcoma [3]. PPSS is a rare variety with only a few cases reported in literature. It usually occurs in the age group of 30-50 years [4] and has equal predilection for males and females [5]. PPSS most commonly presents with chest pain, shortness of breath, hemothorax, and pleural effusion [2]. A case series of 19 PPSS cases indicated that the most common presentation was chest pain accompanied by breathlessness, while the least common presentation was pleural effusion [6]. Further, PPSSs are usually localised, solid pleural tumors but can, less commonly, present with diffuse pleural thickening [2]. Our patient presented with multiple pleural-based lesions with recurrent hemorrhagic pleural effusion.

Depending upon the type of cells, synovial sarcomas are classified histologically into monophasic, biphasic, and poorly differentiated subtypes. In the biphasic type, variable proportions of epithelial and spindle cell components are present. The monophasic type is predominantly composed of spindle cells. The poorly differentiated types show small round tumor cells. Most PPSSs are monophasic [2].

IHC studies reveal that expression of TLE- 1 is a sensitive and specific marker for synovial sarcoma [7]. This was seen in our case as well which, along with CD99 and Pan-CK positivity, led to a final diagnosis of PPSS. Genetic analysis may be helpful in diagnosis when IHC is inconclusive. $\mathrm{t}(\mathrm{X} ; 18)$ (p11.2;q11.2) is the most common chromosomal translocation found in more than $89 \%$ of synovial sarcomas regardless of its histological subtype. In our case, since IHC was confirmatory, genetic studies were not done.

The best treatment for synovial sarcoma is not yet clear. Though many treatment options are available, a multidisciplinary approach involving surgery, chemotherapy, and radiotherapy has been suggested. Radical resection is the first-line treatment, and adjuvant radiotherapy is recommended for cases with incomplete resection. The efficacy of chemotherapy as first line treatment is unclear, however, improvement in overall survival has been described with the use of doxorubicin and ifosfamide. Neoadjuvant chemotherapy could be beneficial for reducing tumor volume and treating micrometastatic disease. Radiofrequency thermal ablation may be considered as an alternative treatment for inoperable patients [8].

\section{Conclusion}

PPSS is a rare disease. Its clinical and histological resemblance to other primary pleural tumors, particularly mesothelioma, can pose a diagnostic challenge. The current case highlights the importance of maintaining a high clinical 

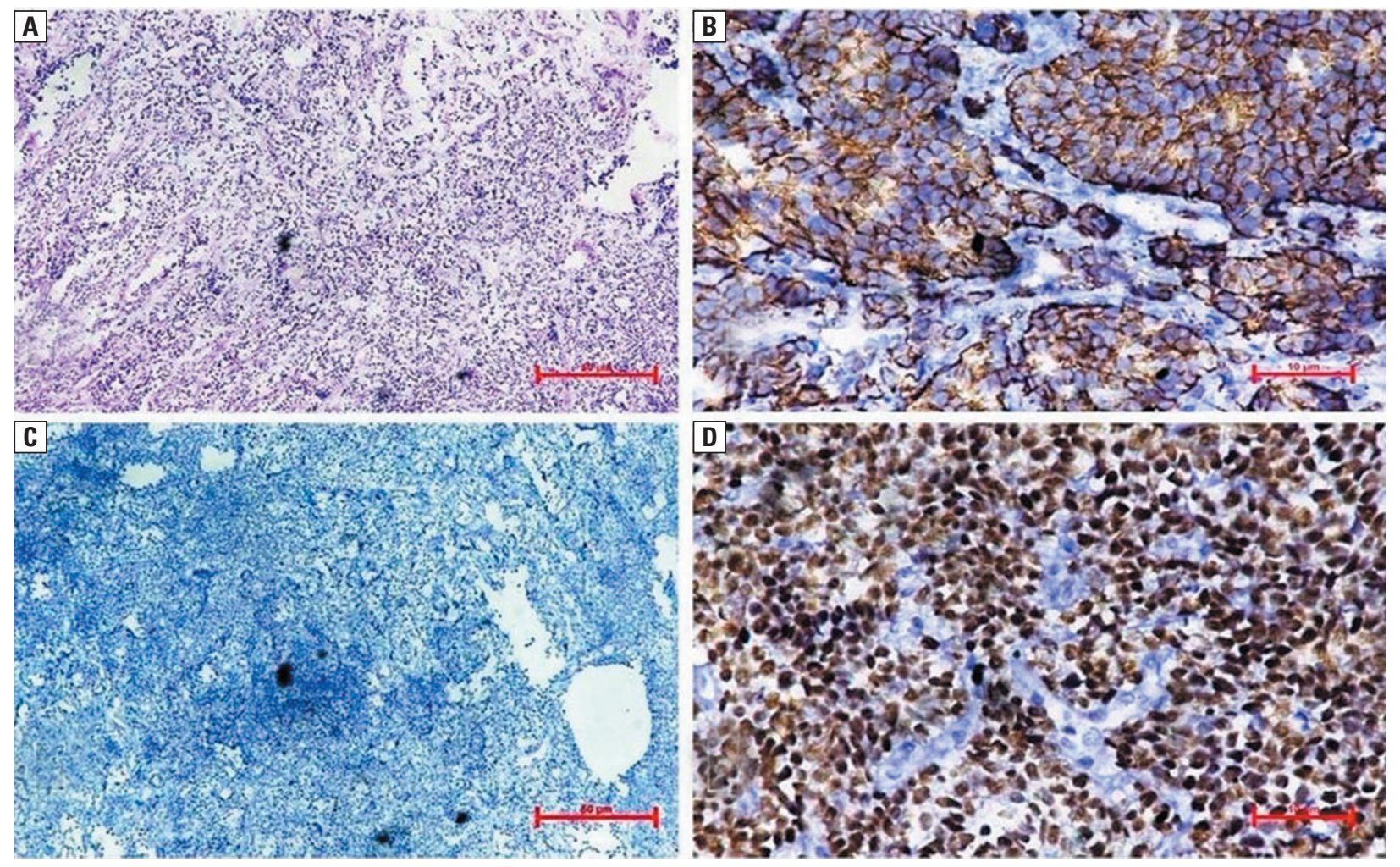

Figure 2. Section from pleural biopsy (H\&E $10 \times$ ) shows small round malignant cells (A). Immunohistochemistry shows nuclear positivity for TLE-1 and membranous positivity for pan-CK (B, D respectively). Tumor cells are negative for calretinin (C)

suspicion of this diagnosis when evaluating pleural tumors presenting with hemorrhagic pleural effusion.

\section{Conflict of interest}

None declared.

\section{References:}

1. Fletcher CDM, Bridge JA, Hogendoorn P, et al. Classification of tumors of soft tissue and bone. Fourth edition. WHO press, Geneva 2013: 213-215.

2. Travis WD, Brambilla E, Burke A, et al. WHO Classification of Tumors of the Lung, Pleura, Thymus and Heart. Fourth edition. WHO press, Geneva 2015: 291.

3. Colwell AS, D’Cunha J, Vargas SO, et al. Synovial sarcoma of the pleura: a clinical and pathologic study of three cases. J
Thorac Cardiovasc Surg. 2002; 124(4): 828-832, doi: $10.1067 /$ mtc.2002.124242, indexed in Pubmed: 12324743 .

4. Tailor J, Roy PG, Bowker C, et al. Primary pleural synovial sarcoma presenting as a multiloculated cyst in an adolescent. Pediatr Surg Int. 2008; 24(5): 597-599, doi: 10.1007/s00383007-2078-4, indexed in Pubmed: 18049822.

5. Mirzoyan M, Muslimani A, Setrakian S, et al. Primary pleuropulmonary synovial sarcoma. Clin Lung Cancer. 2008; 9(5): 257-261, doi: 10.3816/CLC.2008.n.040, indexed in Pubmed: 18824448.

6. Hartel P. Primary pleural synovial sarcoma: clinicopathologic evaluation of 19 cases with focal unusual histology and diagnostic pearls. EC Pulmonology and Respiratory Medicine. 2018; 7: 395-402.

7. Foo WC, Cruise MW, Wick MR, et al. Immunohistochemical staining for TLE1 distinguishes synovial sarcoma from histologic mimics. Am J Clin Pathol. 2011; 135(6): 839-844, doi: 10.1309/AJCP45SSNAOPXYXU, indexed in Pubmed: 21571956.

8. Eilber FC, Dry SM. Diagnosis and management of synovial sarcoma. J Surg Oncol. 2008; 97(4): 314-320, doi: 10.1002 jso.20974, indexed in Pubmed: $\underline{18286474}$. 